\title{
Therapy management in bone sarcoma
}

\author{
Martin Thaler · Ismail Khosravi
}

Received: 5 February 2020 / Accepted: 17 March 2020 / Published online: 15 April 2020

(C) The Author(s) 2020

\begin{abstract}
Summary Bone sarcomas are rare, representing only $0.2 \%$ of all diagnosed cancers. Incidence is higher in children and adolescents, but bone sarcomas are still numerically outnumbered by benign bone tumors in this patient cohort. This article summarizes the management of treatment for bone sarcomas. Systemic therapy, surgical therapy, and radiotherapy are presented. Osteosarcoma, chondrosarcoma, and Ewing sarcoma are among the most frequently occurring bone sarcomas in all age groups. In recent decades, multimodal treatment of these rare entities has increased disease-free survival for these patients. As malignant primary bone tumors are rare cancers, and since management is complex, the standard of treatment should be performed by reference centers. If possible, wide en-bloc resection of the bone sarcoma should be performed. The role of radiotherapy in osteosarcoma and chondrosarcoma is limited, but Ewing sarcoma is a radiation-responsive tumor. In primary bone sarcomas, the efficacy of chemotherapy varies according to histological type. Prognosis is poor in patients with osteosarcoma or Ewing's sarcoma if surgery without neoaqdjuvant chemotherapy is performed. Despite advances in surgical, medical, and radiation therapy, few significant positive changes in overall survival have been observed in patients with these diseases in recent decades.
\end{abstract}

Keywords Malignant bone tumor · Treatment · Surgery $\cdot$ Chemotherapy $\cdot$ Radiation therapy

\footnotetext{
Associate Professor M. Thaler, MD ( $₫) \cdot$ I. Khosravi, MD

Department of Orthopaedic Surgery, Innsbruck Medical University, Anichstraße 35, 6020 Innsbruck, Austria martin.thaler@i-med.ac.at
}

I. Khosravi, MD

ismail.khosravi@tirol-kliniken.at

\section{Introduction}

Malignant bone tumors are rare neoplasms that cause significant morbidity and mortality. Therefore, these patients require optimal treatment. The most common bone sarcomas include osteosarcoma, Ewing's sarcoma, and chondrosarcoma. Accordingly, bone sarcomas are uncommon and represent only $0.2 \%$ of all malignancies [1]. However, they are highly aggressive and their treatment is extremely challenging. Approximately 3020 bone sarcomas are newly diagnosed annually in the United States, resulting in 1460 deaths each year [1]. The adjusted incidence rate for all bone and joint malignancies is 0.9 per 100,000 persons per year. Sarcomas of the bone can be found in all age groups. However, in many cases, a relationship between the patient's age and the specific location and type of tumor can be observed. Bone sarcomas have two peaks regarding their age distribution. The first peak develops in patients aged 10-20, while the second peak occurs in the seventh decade of life. Bone sarcomas are outnumbered by benign bone tumors in the first age peak and by bone metastases in the second age peak. More bone sarcomas are diagnosed in the second age peak [2]. They are predominantly located at the pelvis, axial skeleton, and long bones, very rarely affecting small bones of the hands and feet [2]. The ratio between male and female patients is 1:0.7. This article presents the current treatment approach for patients with bone sarcomas.

\section{Treatment}

The majority of patients with bone sarcomas require a multidisciplinary approach that includes orthopedic, medical and radiation oncologists, plastic surgeons, pathologists, as well as radiologists with expertise in bone tumors. Surgery is the primary ther- 
Table 1 Classification of primary bone sarcomas

\begin{tabular}{|c|c|}
\hline Type & Subtype \\
\hline \multirow[t]{5}{*}{ Chondrosarcoma } & Conventional \\
\hline & Dedifferentiated \\
\hline & Clear cell \\
\hline & Mesenchymal \\
\hline & Secondary \\
\hline \multirow[t]{9}{*}{ Osteosarcoma } & Conventional \\
\hline & Chondroblastic, fibroblastic, osteoblastic, mixed \\
\hline & Parosteal \\
\hline & Periosteal \\
\hline & Intramedullary well differentiated \\
\hline & Surface high grade \\
\hline & Small cell \\
\hline & Telangiectatic \\
\hline & Secondary \\
\hline \multicolumn{2}{|l|}{ Fibrosarcoma } \\
\hline \multicolumn{2}{|c|}{ Ewing sarcoma and other round cell sarcomas } \\
\hline \multicolumn{2}{|l|}{ Chordoma } \\
\hline \multirow{4}{*}{$\begin{array}{l}\text { Malignant vascular } \\
\text { tumors }\end{array}$} & Epithelioid hemangioendothelioma \\
\hline & Pseudomyogenic hemangioendothelioma \\
\hline & Angiosarcoma \\
\hline & Kaposi sarcoma \\
\hline \multirow{6}{*}{$\begin{array}{l}\text { Other uncommon } \\
\text { entities }\end{array}$} & Adamantinoma \\
\hline & Liposarcoma \\
\hline & Leiomyosarcoma \\
\hline & Malignant peripheral nerve sheath tumor \\
\hline & Primary non-Hodgkin lymphoma \\
\hline & Synovial sarcoma \\
\hline
\end{tabular}

apeutic approach. The role of chemotherapy and radiation therapy is individualized. The classification of bone sarcomas is based on cell and tissue type, specific histological characteristics, relationship to the bone, and biological potential. Table 1 shows the most widespread classification system, which is provided by the World Health Organization. For some bone sarcomas, such as osteosarcoma and Ewing's sarcoma, there is a preference to treat the patient with adjuvant chemotherapy prior to surgical treatment, thereby aiming to treat potential micrometastatic disease, reduce the soft tissue mass of the bone tumor, and/or mature the mass, allowing easier resection [3, 4].

\section{Radiotherapy}

The role of radiation therapy is individualized. Radiation therapy can be administered for local tumor control in patients in whom bone sarcomas are located in difficult locations, or where a wide resection of the tumor would have a significant impact on the patient's ability to live (spine, head, pelvis [5]). It has been proven that Ewing sarcoma is a radiation-responsive tumor. Radiation therapy can be used in Ewing sarcomas in small tumors, tumors that are not resectable, in the palliative setting, and in a multidisciplinary approach. Chondrosarcomas, osteosarcomas, and chordomas are not radiation-sensitive [6]. The doses for chondrosarcomas, osteosarcomas, and chordomas to achieve local control of the disease vary from $66 \mathrm{~Gy}$ to $>75$ Gy. Radiation therapy can be used preoperatively, intraoperatively, and postoperatively. This depends on the site and type of tumor, the achieved surgical resection margins, and the response to chemotherapy [6]. In cases of difficult locations of sarcomas, chemotherapy can be used to reduce and limit tumor margins. Intraoperative and postoperative radiation therapy is used for patients with inadequate resection margins, pathological fractures, poor response to chemotherapy, or inappropriate surgical margins [7]. In rare cases, primary radiation therapy can be used for bone sarcomas if a complete wide resection is either technically not possible or resection is unacceptable for the patient. It can also be used in palliative cases [8]. For example, chondrosarcomas in the skull are often not resectable. In such cases, proton beam radiotherapy should also be considered [8].

\section{Chemotherapy}

Chemotherapy for bone sarcomas is usually administered in a neoadjuvant scheme. The advantages are that this provides demarcation of the tumor prior to surgery and histological tumor response can be assessed after resection of the tumor. Since the incidence of bone sarcomas is so rare, it is recommended that medical oncologists register bone sarcoma patients in international cooperative group trials. For male patients at a reproductive age, sperm storage can be offered. The options regarding reproduction for female patients should be discussed with a fertility expert prior to therapy.

Osteosarcoma and Ewing sarcoma are chemotherapy-sensitive tumors. Most chondrosarcoma subtypes do not respond to chemotherapy. Recent reports postulate that mesenchymal chondrosarcoma may be chemotherapy-sensitive and may be considered for adjuvant or neoadjuvant therapy. There is some doubt as to whether dedifferentiated chondrosarcoma and mesenchymal chondrosarcoma are chemotherapy sensitive, but they are often treated like osteosarcoma [8]. For the curative treatment of high-grade osteosarcoma, a combination of surgery and chemotherapy is necessary. Compared with surgery alone, multimodal treatment of high-grade osteosarcoma increases disease-free survival from $10-20 \%$ to $>60 \%$. In most cases, the protocols include a period of preoperative chemotherapy, although hard evidence on the benefit regarding survival over postoperative chemotherapy alone is lacking [5].

Current treatment regimens for Ewing sarcomas include neoadjuvant chemotherapy, followed by surgery 
and adjuvant chemotherapy. This protocol leads to cure in approximately two-thirds of patients with localized disease [9].

If Ewing sarcomas are treated with surgery or radiotherapy alone, 5-year survival is $<10 \%$. The multimodality treatment regime with chemotherapy increases survival rates to $60-70 \%$ in localized disease and to $20-40 \%$ in metastatic disease [8].

Chemotherapy in Ewing sarcoma is used according to the EURO E.W.I.N.G. 99 (European Ewing Tumour Working Initiative of National Groups) protocol. Chemotherapy in osteosarcomas is administered in younger patients according to the EUARMOS (European and American osteosarcoma study group) protocol or the EURO BOSS (European bone over 40 sarcoma study) trial in patients older than 40 years [9, 10].

In general, poor histological response to preoperative chemotherapy is a negative prognostic factor.

\section{Surgery}

\section{Resection}

The first step in tumor resection is to perform an adequate biopsy of suspected bone in accordance with the planned resection of the bone sarcoma. The biopsy of a suspected bone sarcoma should be carried out at a reference center and performed or supervised by a physician experienced in limb salvage for bone sarcomas. In ideal circumstances, the surgeon that will perform the tumor resection should perform the biopsy $[11,12]$. The goal of biopsy is to obtain diagnostic tissue while minimizing morbidity, limiting potential tumor spread, and avoiding interference with future treatments [13].

Surgery should only be performed after adequate preoperative staging as well as radiological evaluation with recent computed tomography and magnetic resonance imaging of the affected tumor site, and, depending on the tumor entity, neoadjuvant chemotherapy should be performed. The ultimate goal of surgery is wide en-bloc resection of the tumor and to provide microscopically tumor free margins [9]. This can be done in an intracompartmental approach, but in the case of clear indications (easily removable bone, muscle), the entire bone/muscle compartment (extracompartmental) can be removed. The tumor with the biopsy scar needs to be removed with an unviolated cuff of $2-3 \mathrm{~cm}$ of healthy tissue. In high-grade sarcomas, the aim of surgery is to avoid entering the reactive zone (possibly microscopically positive) of the tissue surrounding the tumor [5]. Intralesional resection: if the tumor is cut or opened at any time during surgery; marginal resections: to be avoided if a cuff of less than $2-3 \mathrm{~cm}$ is left on the tumor (microscopically positive). Both are postulated to have a higher local recurrence rate and an adverse effect on overall survival [14]. For low-grade chondrosarcomas of the extremities, intralesional surgery is acceptable, since this has been reported to have a low rate of recurrence [15].

The goal of surgery is to safely remove the tumor and to preserve as much function as possible. Amputation was the standard surgical treatment in the case of bone sarcomas 40-50 years ago. Nowadays, most patients should be considered candidates for limb salvage, with the indication for amputation becoming rare. Amputation can be considered if curative treatment with limb salvage is not possible. Amputation may be required if the tumor invades major vessels and nerves, intraoperative negative resection margins are obtained, or a functionally insufficient extremity is the result of surgery [16].

If neurovascular structures are not surrounded by the sarcoma, these structures can be spared. If more than $50 \%$ of the neurovascular structures are encased by the sarcoma, arterial resection may be performed to bypass the vessel and leave the encased structure on the resected tumor [8]. In these cases, reverse interpositional vein graft, synthetic graft, or vein allograft can be used to restore vascular function.

\section{Fractures}

In cases of pathological fracture caused by osteosarcoma, neoadjuvant chemotherapy should be used in the expectation that a good response will allow the fracture hematoma to retract and allow subsequent resection of the tumor and the involved soft tissues. Published data showed that surgery followed by neoadjuvant chemotherapy could boost the overall survival rate to $92 \%$, in contrast to $58 \%$ of patients receiving manipulative therapy. Patients that did not receive neoadjuvant chemotherapy had significantly higher rates of metastasis and poorer prognosis $(p<0.05)[17,18]$. In patients with a poor response to chemotherapy or in tumors that are not sensitive to chemotherapy, like chondrosarcomas, early surgery obtaining wide margins should be considered. In some cases, this may require amputation of the affected extremity.

\section{Reconstruction}

Following limb salvage, bone and soft tissue reconstruction is necessary. Megaprosthetic or allograft reconstruction can be performed. Allograft prosthesis composites are frequently used in certain tumor locations like the proximal humerus or the proximal tibia. Biological reconstruction, such as the implantation of allografts or vascularized and non-vascularized fibulas, is mainly used for the interposition of diaphyseal defects [19]. In the majority of patients, modular megaprosthetic reconstruction is used for limb salvage. Some tumor locations are highly challenging for reconstruction. For example, pelvic resection and reconstructions are one of the most challenging pro- 
cedures. Pelvic resections often include resections of the sacrum or the spine, and these structures are very difficult to reconstruct.

In growing children, reconstruction after bone sarcoma resection is challenging, particularly in terms of addressing leg length inequality. If resection involves the joint, an expandable prosthesis or rotationplasty should be considered [20].

\section{Take home message}

- Bone sarcomas are rare, but appropriate treatment is crucial and requires an interdisciplinary team of surgeons, oncologists, and radiotherapists.

- All patients should receive individual specialist treatment in reference centers with the aim of limb salvage.

Funding Open access funding provided by University of Innsbruck and Medical University of Innsbruck.

Conflict of interest M. Thaler and I. Khosravi declare that they have no competing interests. None of the authors has received support for this work from any of the following organizations: National Institutes of Health (NIH), Welcome Trust, Howard Hughes Medical Institute (HHMI), or other foundation(s) requiring open access.

Open Access This article is licensed under a Creative Commons Attribution 4.0 International License, which permits use, sharing, adaptation, distribution and reproduction in any medium or format, as long as you give appropriate credit to the original author(s) and the source, provide a link to the Creative Commons licence, and indicate if changes were made. The images or other third party material in this article are included in the article's Creative Commons licence, unless indicated otherwise in a credit line to the material. If material is not included in the article's Creative Commons licence and your intended use is not permitted by statutory regulation or exceeds the permitted use, you will need to obtain permission directly from the copyright holder. To view a copy of this licence, visit http://creativecommons.org/licenses/by/4.0/.

\section{References}

1. Siegel R, Ma J, Zou Z, Jemal A. Cancer statistics, 2014. CA Cancer JClin. 2014;64(1):9-29. https://www.ncbi.nlm.nih. gov/pubmed/24399786.

2. Dorfman HD, Czerniak B. Bone cancers. Cancer. 1995;75(1 Suppl):203-10. https://www.ncbi.nlm.nih.gov/pubmed/ 8000997.

3. Mavrogenis AF, Angelini A, Pala E, Calabro T, Bianchi G, Casadei R, et al. Radiation-induced sarcomas. J Long Term Eff Med Implants. 2011;21(3):233-40.

4. Mavrogenis AF, Pala E, Guerra G, Ruggieri P. Post-radiation sarcomas. Clinical outcome of 52 Patients. J Surg Oncol. 2012;105(6):570-6.

5. Ozaki T, Flege S, Kevric M, Lindner N, Maas R, Delling G, et al. Osteosarcoma of the pelvis: experience of the Cooperative Osteosarcoma Study Group. J Clin Oncol. 2003;21(2):334-41.
6. Patel S, DeLaney TF. Advanced-technology radiation therapy for bone sarcomas. Cancer Control. 2008;15(1):21-37.

7. Hug EB. Review of skull base chordomas: prognostic factors and long-term results of proton-beam radiotherapy. Neurosurg Focus. 2001;10(3):E11-E11.

8. Mavrogenis AF, Angelini A, Vottis C, Palmerini E, Rimondi E, Rossi G, et al. State-of-the-art approach for bone sarcomas. Eur J Orthop Surg Traumatol. 2014;25(1):5-15.

9. Bernstein M, Kovar H, Paulussen M, Randall RL, Schuck A, Teot LA, et al. Ewing's sarcoma family of tumors: current management. Oncologist. 2006;11(5):503-19.

10. Bielack SS, Machatschek J-N, Flege S, Jürgens H. Delaying surgery with chemotherapy for osteosarcoma of the extremities. Expert Opin Pharmacother. 2004;5(6):1243-56.

11. Bickels J, Jelinek JS, Shmookler BM, Neff RS, Malawer MM. Biopsy of musculoskeletal tumors. Current concepts. Clin Orthop Relat Res. 1999;368:212-9.

12. Mankin HJ, Mankin CJ, Simon MA. The hazards of the biopsy, revisited. Members of the musculoskeletal tumor society. JBone Joint SurgAm. 1996;78(5):656-63.

13. Kasraeian S, Allison DC, Ahlmann ER, Fedenko AN, MenendezLR.A comparison offine-needleaspiration, corebiopsy, and surgical biopsy in the diagnosis of extremity soft tissue masses. Clin Orthop Relat Res. 2010;468(11):2992-3002.

14. Enneking WF. A system of staging musculoskeletal neoplasms. Clin Orthop Relat Res. 1986;204:9-24.

15. Mavrogenis AF, Angelini A, Drago G, Merlino B, Ruggieri P. Survival analysis of patients with chondrosarcomas of the pelvis. J Surg Oncol. 2013;108(1):19-27.

16. Jawad MU, Scully SP. In brief: classifications in brief: enneking classification: benign and malignant tumors of the musculoskeletal system. Clin Orthop Relat Res. 2010;468(7):2000-2.

17. Kelley LM, Schlegel M, Hecker-Nolting S, Kevric M, Haller B, Rössig C, et al. Pathological fracture and prognosis of highgrade osteosarcoma of the extremities: an analysis of 2,847 consecutive cooperative osteosarcoma study group (COSS) patients. J Clin Oncol. 2020;38(8):823-33. https://doi.org/ 10.1200/JCO.19.00827.

18. Chung LH, Wu PK, Chen CF, Weng HK, Chen TH, Chen WM. Pathological fractures in predicting clinical outcomes for patients with osteosarcoma. BMC Musculoskelet Disord. 2016; https://doi.org/10.1186/s12891-016-1351-x.

19. Ruggieri P, Mavrogenis AF, Casadei R, Errani C, Angelini A, Calabrò $\mathrm{T}$, et al. Protocol of surgical treatment of long bone pathological fractures. Injury. 2010;41(11):1161-7.

20. Ruggieri P, Mavrogenis AF, PalaE, Romantini M, Manfrini M, Mercuri M. Outcome of expandable prostheses in children. JPediatr Orthop. 2013;33(3):244-53.

Publisher's Note Springer Nature remains neutral with regard to jurisdictional claims in published maps and institutional affiliations.

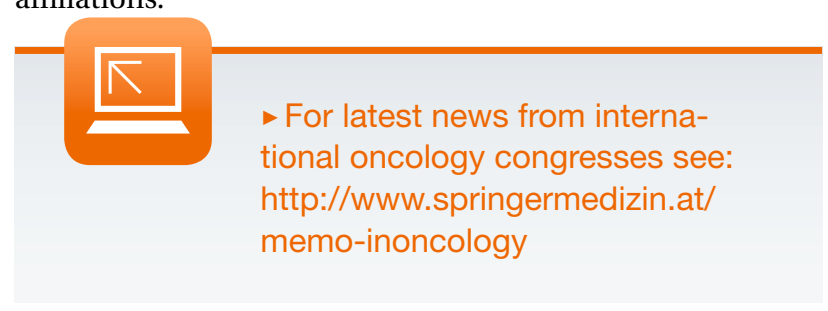

